
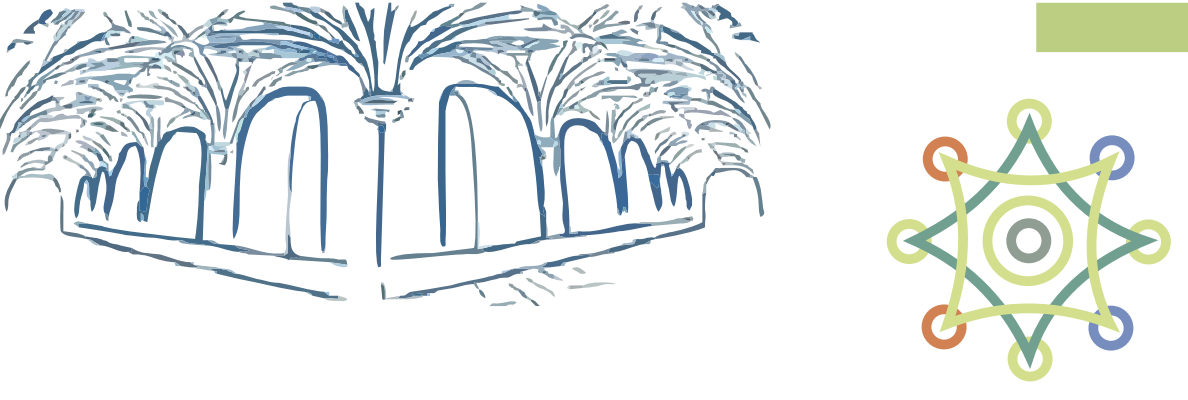

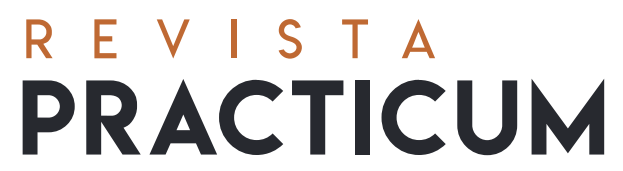

Número 6(1), Enero-Junio, 2021

ISSN: 2530-4550

Guedes, A., Antunes, S., Santos, P., Oliveira, I.

Fecha de recepción: 30/04/2020

\& Escola, J. (2021).

Fecha de aceptación 17/09/2020

Os desafios da avaliação e os Portefólios.

Revista Practicum, 6(1), 95-109.

https://doi.org/10.24310/RevPracticumrep.v6i1.10160

\title{
Os desafios da avaliação e os Portefólios
}

\section{Assessment Challenges \& Portfolios}

\section{Desafíos en la evaluación y Portafolios}

\author{
Anabela Guedes \\ IPV-ESTGL \\ aguedes@estgl.ipv.pt \\ Sandra Antunes \\ IPV-ESTGL \\ santunes@estgl.ipv.pt \\ Paula Santos \\ IPV-ESTGL \\ psantos@estgl.ipv.pt \\ Isabel Oliveira \\ IPV-ESTGL \\ ioliveira@estgl.ipv.pt \\ José Escola \\ UTAD \\ jescola@utad.pt
}

\section{Resumo}

Os portefólios podem ser definidos como a retenção e organização de instrumentos de informação sobre os processos realizados pelo aluno durante um de- 
terminado período, os quais que podem fornecer uma visão ampla e o mais detalhada possível dos diferentes componentes afetivos cognitivos, metacognitivos e afetivos, e até desenvolvimento moral. Este artigo tem como objetivo abordar a relevância do uso de portefólios por professores que favorecem a reflexão, a autonomia na aprendizagem e a inovação no processo de ensino-aprendizagem. $\mathrm{Na}$ Educação, o portefólio assumiu um papel importante e tornou-se numa ferramenta de avaliação, que dá visibilidade ao conhecimento aprendido, o que leva à reflexão, que destaca os diferentes elementos do desenvolvimento do aluno e, portanto, não é apenas uma amostra de atividades.

\section{Resumen}

Los portafolios pueden definirse como la retención y organización de instrumentos de información sobre los procesos llevados a cabo por el alumno durante un determinado período y que pueden proporcionar una visión amplia y lo más detallada posible de los diferentes componentes cognitivos, metacognitivos, afectivos e incluso desarrollo moral. Este artículo tiene como objetivo abordar la relevancia del uso de portafolios por parte de maestros que favorecen la reflexión, la autonomía en el aprendizaje y la innovación en el proceso de enseñanza-aprendizaje. En Educación, el portafolio ha asumido un papel importante y se ha convertido en una herramienta de evaluación, que da visibilidad al conocimiento aprendido, lleva a la reflexión, resalta los diferentes elementos del desarrollo de los estudiantes y, por lo tanto, no es solo una muestra de actividades.

\section{Abstract}

Portfolios can be defined as retaining and organizing information instruments concerning the processes carried out by the student over a certain period and which are able to provide a broad view and as detailed as possible of the different components of its cognitive, metacognitive, affective and even moral development. This article aims to address the relevance of using portfolios by teachers who favour reflection, autonomy in learning, and innovation in the teaching-learning process. In Education, the portfolio has taken an important role, and has become an assessment tool, which gives visibility to the knowledge learned, leads to reflection, highlights the different elements of students' development and it is not, therefore, just a sampling of activities.

\section{Palabras claves}

Portafolios, evaluación formativa, constructivismo, enfoque reflexivo, autoevaluación. 


\section{Keywords}

Portfolios, formative assessment, constructivism, reflexive approach, self-assessment.

\section{Palavras-chave}

Portefólios, avaliação formativa, construtivismo, enfoque reflexivo, autoavaliação.

\section{Introduçao}

A incessante procura do professor pelo método perfeito para ensinar é tão fascinante e equiparável à sua conformação (não menos inquietante) pelo instrumento perfeito para avaliar.

No que diz respeito ao ensinar, chegou-se a acreditar que era possível a descoberta de um método que pudesse ser bem-sucedido em todos os contextos e com todos os alunos (Nunan, 1995), sendo que a constante procura pelo método perfeito foi durante muito tempo uma obsessão (Brown, 2001). Foram necessárias experiências com muitos métodos de ensino para que se procurasse, não o método perfeito, mas sim, o método mais adequado (Duque, 2004). Todavia, ainda hoje, quando começa mais um ano letivo, o professor se debate com o questionamento constante de encontrar a melhor maneira para ensinar aquele conteúdo.

Já no que diz respeito ao avaliar, a insistência por instrumentos de avaliação sumativos que quantificam e ordenam os resultados obtidos dos alunos tem vigorado na maioria dos estabelecimentos de ensino, sendo que é bem notória a negligência que se atribui à diversidade de percursos e ao historial dos alunos, assim como os diversos tipos de inteligência que possuem, pelo que utilizar repetidamente os mesmos instrumentos de avaliação beneficia sempre aqueles que mais se identificam com esse tipo de técnicas (Neves \& Barbosa, 2006).

A utilização de um instrumento como o portefólio que, pela sua natureza, implica o desenvolvimento de aspetos metacognitivos, é uma das respostas possíveis que o professor tem à sua disposição para dar resposta ao desafio primordial aquando do processo de ensino que é o de ensinar os alunos a pensar. Este objetivo, em concordância com teorias atualizadas de ensino/aprendizagem e de avaliação formativa, assentam em determinadas conviç̧ões ou em teorias integradas e interrelacionadas, nomeadamente nas ideias subjacentes às perspetivas do construtivismo, nas quais se pressupõe que cada aluno aprende e atribui significado ao que o rodeia, ligando o que já sabe e experimenta ao que está a aprender, sobretudo quando lhe são colocadas questões relevantes, encorajan- 
do, assim, a reflexão crítica e o autoquestionamento, valorizando o seu ponto de vista e avaliando a aprendizagem de forma contextualizada e continuada.

\section{Metodologia}

Este trabalho segue uma metodologia humanístico-interpretativa de cariz essencialmente descritivo, considerados os seus objetivos. Esta metodologia é particularmente aquela que subjaz a estudos exploratório-descritivos, combinando a recolha de dados secundários.

Numa investigação sobre a educação, a compreensão do fenómeno educativo é o seu objetivo maior, pelo que a decisão sobre a escolha da metodologia apropriada é sempre da máxima importância.

Movidas pela conviç̧ão de que nos segundos ciclos há uma obrigatoriedade de refletir, ainda mais profundamente, em relação aos instrumentos a serem usados para aferir a aquisição das competências previamente estabelecidas para as unidades curriculares, levámos a cabo esta investigação que pretendia, pela leitura de artigos científicos e análise dos resultados de outras investigações sobre a temática, conhecer o estado da arte e avançar com algumas proposições que respondam às questões que nos mobilizam.

O estudo assentou numa exploração bibliográfica preferencialmente orientada para a recolha de dados em artigos e estudos científicos realizados em torno dos conceitos de avaliação e do instrumento de avaliação chamado de portefólio, sujeitos a procedimentos de análise de conteúdo (de tipo qualitativo, direta e de exploração) e de análise argumentativa.

\section{Contexto}

\subsection{0 ensino superior}

O quadro geral do sistema educativo Português está definido na Lei de Bases do Sistema Educativo (Lei № 46/86, de 14 de outubro, posteriormente alterada, nalguns dos seus articulados pelas Leis nos 115/97, de 19 de setembro, e 49/2005, de 30 de agosto, republicada e renumerada em anexo à última) e é o referencial normativo das políticas educativas que visam o desenvolvimento da educação e do sistema educativo no território Português. No que diz respeito ao ensino superior, em 2005 foram dados os primeiros passos para a reforma do sistema de ensino superior, com a introdução de um novo sistema de créditos (ECTS - european credits transfer system) para ciclos de estudo e, neste ano, foram efetuadas alterações à Lei de Bases do Sistema Educativo de modo a permitir a implementação do Processo de Bolonha. Esta nova estrutura contem- 
pla três ciclos de estudo e foi introduzida em 2006, embora apenas totalmente implementada, em Portugal, a partir do ano letivo de 2009/2010. As muitas e substanciais mudanças que ocorreram desde então no panorama do ensino superior, com forte impacto no sistema educativo nacional, conduziram a que $o$ ensino superior Português seja, desde então, estruturado ao abrigo dos princípios da Declaração de Bolonha e é ministrado, quer em institutos politécnicos, quer em universidades (dada a existência de um sistema binário no ensino superior, em Portugal).

Para este trabalho, interessa-nos que nos foquemos no segundo ciclo de estudos (mestrados) e no quadro nacional de qualificações que estabelece os resultados de aprendizagem correspondentes. Assim, na página da Direção Geral do Ensino Superior (www.dges.gov.pt/pt/quadro_qualificacoes) e no que diz respeito aos conhecimentos, o mesmo estabelece que os segundos ciclos devem conduzir o aluno à obtenção de "conhecimentos altamente especializados (...) numa determinada área de estudo ou de trabalho, que sustentam a capacidade de reflexão original e ou investigação." Ainda sobre conhecimentos, definiu-se que este ciclo de estudos deve conduzir o aluno a desenvolver uma "consciência crítica das questões relativas aos conhecimentos". Já no campo das atitudes, pretende-se que o aluno, no final da frequência do ciclo de estudos tenha a capacidade de "gerir e transformar contextos de estudo (...)" e "assumir responsabilidade por forma a contribuir para os conhecimentos".

Este introito sobre a organização do ensino superior e sobre os resultados de aprendizagem estabelecidos tem como objetivo relacionar a necessidade de pensar em formas de avaliação que vão ao encontro dos resultados de aprendizagem preconizados e centrar a nossa reflexão em como nós, enquanto agentes educativos, concebemos as unidades curriculares de maneira a contribuirmos para que os alunos sejam conduzidos a alcançar quer os conhecimentos quer as atitudes traçadas para o ciclo de estudos que frequentam.

\subsection{As escolas e os professores}

A escola assume, desde sempre, um papel único e primordial de integração de saber e tem a "obrigação" permanente de se adaptar às constantes mudanças societais.

Nas escolas, em geral, e no ensino superior, em particular, verifica-se, por vezes, um predomínio da componente "académica", compartimentada, hierarquizada, abstrata, teórica e dedutiva (Formosinho, 1992) com um currículo planeado centralmente e uniformemente para todos. Estas características arrastam consigo uma pedagogia uniforme e a relação pedagógica assenta, essencialmente, na autoridade e figura do professor como garante da verdade e possui, por vezes, 
um conhecimento pouco dinâmico, tornando o processo de ensino/aprendizagem um processo teórico e unidirecional.

Para que consiga a concretização da troca de saberes e a interação social, é necessário que o professor tenha noção do papel que lhe é conferido, das responsabilidades que tem de enfrentar quando transmite conhecimento e, sobretudo, do estatuto de aluno que irá receber. É necessário que, logo à partida, reconheça que os métodos e instrumentos de avaliação que terá de integrar no processo que medeia podem proporcionar um espaço de profunda renovação. Por isso, é essencial uma mudança qualitativa nos processos de ensino e aprendizagem, com repercussões nos níveis organizativo, de conteúdo, metodológico e na aprendizagem.

É imperativo, ainda, que o professor se questione e verifique se há algum descompasso entre o tipo de acesso ao conhecimento que o aluno adquire fora da escola e o conhecimento que o professor transmite na sala de aula. Tal como nos refere Matos (2006), com o conhecimento adquirido fora da escola, o aluno vivencia as situações, relaciona-as e espontaneamente as transforma em aprendizagens significativas. É bem óbvio que o nosso tempo está muito mais próximo deste paradigma do que daquele em que assenta a tradicional metodologia de ensino/aprendizagem vigente em algumas das nossas escolas. Neste sentido, precisamos que o professor assuma um papel reflexivo, estratégico e investigador, sem, conquanto, deixar de ter um papel fulcral e essencial no aconselhamento e acompanhamento, ou seja, na formação do aluno (Matos, 2006).

Nesta conjuntura, o professor deixa de ser o orador que todos nos habituámos, para passar a ser o mediador, já que a tónica se coloca na conquista do conhecimento e da sabedoria, com base no trabalho de equipa ou na cooperação, assumindo-se o professor como como guia e conselheiro no acesso e seleção da informação, bem como de decisor das estratégias plausíveis a serem pontualmente desencadeadas ou, mesmo, como co-aprendiz (cf. "professor-aprendiz" in Leite, 1995). Com base nisto, e face ao contexto escolar e societal, é natural que assistamos a uma maleabilidade das suas atuações. Os alunos requerem, portanto, que o processo de transmissão do conhecimento seja continuamente pensado e reinventado de acordo com as características que constantemente assimilam e que Ihes são incutidas pela própria evolução da sociedade. Norteados pelo pensamento que é na Educação que reside a importância e toda a potencialidade do desenvolvimento humano e societal, a escola não pode nunca deixar de se preocupar em desenvolver paradigmas de ensino-aprendizagem que fomentem uma aproximação às necessidades formativas dos alunos e ao desenvolvimento de didáticas que tenham em conta o perfil do sujeito que é alvo desse ensino. Para isso, a educação precisa, reiteradamente, de rever aspetos basilares dos seus fundamentos teórico-metodológicos, abrindo caminhos para 
aprendizagens cooperativas, formando uma inteligência coletiva que se constrói e reconstrói através do diálogo dos saberes, integrando todas as dimensões do ser-humano (Moran, 2000).

Perante todos estes argumentos podemos, não só aferir e compreender os obstáculos que condicionam e manietam os agentes educativos como facilitadores do processo educativo, mas também comprovar a necessidade de mudança de mentalidade e atitude por parte de quem ensina. É obrigatória uma disponibilidade mental dos docentes em alterarem as suas metodologias e técnicas de ensino, o que obriga a uma "revolução" educativa, às vezes anual, sempre que é chamado a repensar em modos de avaliação que sejam um fator de aproximação professor-aluno e não um móbil de afastamento, isolando o professor das exigências societais e educativas contemporâneas.

Acreditamos que o futuro perspetivado da sociedade depende significativamente do que ocorre, sempre, nas escolas, sendo que as características e a qualidade da ação educativa que aí decorre, as aprendizagens realizadas, as competências e os saberes adquiridos são fatores condicionantes do percurso social a realizar. Nesta sociedade que se nos impõe, é imperativo que os alunos sejam capazes de incrementar, de forma flexível e faseada, processos de aprendizagem transdisciplinar que lhes garanta a transferibilidade das aprendizagens e a autonomia que se pretende. Para isto, é inequívoco o empenho das escolas e dos professores para o estímulo de aprendizagens autónomas e cooperativas dos e com os alunos para que seja assegurado um percurso coerente de formação e a aquisição de um conjunto de competências claramente referenciadas e adequadas às exigências societais e às competências de cada nível de formação.

\section{Avaliação das Aprendizagens}

Há duas palavras estruturantes no âmbito da avaliação de aprendizagens que é necessário, de imediato, ressalvar antes de se explanar e discorrer sobre o assunto que são as palavras sumativa e formativa. Estas duas palavras prevalecem no contexto educativo desde que, em 1967, foram usadas por Michael Scriven, sendo que, contudo, a sua concetualização tem evoluído desde essa altura. Como já adiantamos, Michael Scriven propôs os termos formativo e sumativo em 1967 para explicar os dois papéis distintos que a avaliação poderia desempenhar na avaliação do currículo. Nos anos seguintes, Benjamin Bloom (1969) e na obra Handbook on the formative and summative evaluation of student learning, em 1971, sugeriu a aplicação da mesma distinção à avaliação da aprendizagem dos alunos, o que hoje tendemos, como William (2006) assevera, a chamar de avaliação.

Como avocámos anteriormente, nas escolas em geral e no ensino superior, em particular, verifica-se, por vezes, a predominância da componente "académica", 
abstrata, teórica e dedutiva e a avaliação das aprendizagens fazia-se (e ainda se faz), muitas vezes, com um exame escrito que contempla os conteúdos lecionados durante um semestre inteiro e cuja classificação se reflete na nota final que o estudantes obtêm à unidade curricular. A corroborar o que anteriormente referimos, num artigo denominado Avaliação das aprendizagens no ensino superior. Perceções de professores e estudantes nas universidades portuguesas, os autores verificaram que:

professores e estudantes percepcionam as práticas de avaliação como essencialmente sumativas, mais os estudantes do que os professores, reconhecendo estes últimos, mais potenciais formativos a estas mesmas práticas (Barreira et al., 2017, p. 32).

Em jeito sumário, apresentamos as duas perspetivas existentes sobre o papel da avaliação no processo de ensino e aprendizagem:

Avaliação sumativa: concentra-se nos resultados educacionais e identifica-se, fundamentalmente, com a função de classificação, de confirmação de saberes adquiridos que se refletem na seriação dos alunos atribuindo-lhes uma posição numa determinada escala (Brown \& Gerhardt, 2002; Perrenoud, 1998).

Avaliação formativa: tem como função a regulação do ensino e da aprendizagem, permitindo apurar se os alunos estão a realizar os progressos pretendidos e propõe-se encontrar os caminhos necessários para que consigam atingir os objetivos de aprendizagem almejados (Lopes \& Silva, 2010, 2012; Silva \& Lopes, 2015). Acresce que a avaliação formativa e formadora (Nunziati, 1990) citada por Prazeres \& Casanova (2015), permite que se façam apreciações e comentários de modo a responder ao compromisso de alterar o que é insatisfatório.

Atualmente, está à disposição do professor uma grande variedade de estratégias, ferramentas e recursos para o apoiar a aperfeiçoar a sua capacidade para aferir o desempenho dos alunos e ajustar a sua prática para ir ao encontro das necessidades dos alunos e de melhorar, assim, os resultados de aprendizagem. Desde logo, há um pressuposto generalizado na literatura da especialidade de que a avaliação formativa leva a melhores resultados de aprendizagem, o que, por si só, é de imediato, relevante.

\subsection{A perspetiva construtivista}

Para que se compreenda como se aprende, é necessário que o professor conheça o que acontece aquando de um processo de ensino/aprendizagem para que, com isso, consiga de uma maneira mais eficaz, orientar a sua ação educativa. Embora nem sempre quem ensina saiba descrever com exatidão a teoria sobre a qual assenta a sua prática pedagógica, a verdade é que o seu conceito de 
aprendizagem e o seu posicionamento teórico estão sempre bem patentes quando são traçados os objetivos e quando são definidas as técnicas que serão usadas na ação educativa. Para este trabalho, foi importante recordarmos a teoria construtivista da aprendizagem, já que nos fornece dados pertinentes que permitem retirar ilações/implicações para as práticas educativas. Mesmo não sendo, como atesta Fostnot (1992) uma teoria do ensino, é uma teoria do conhecimento e de aprendizagem que tem tido uma grande influência no domínio educativo (Bidarra \& Festas, 2005) e proporciona uma epistemologia alternativa às tradições objetivas do conhecimento, da aprendizagem e do ensino.

A aprendizagem construtivista é baseada na participação ativa dos alunos na solução de problemas e no pensamento crítico sobre uma atividade de aprendizagem que eles consideram relevante e envolvente. São os alunos que constroem o seu próprio conhecimento, testam ideias e abordagens com base no seu conhecimento e experiências anteriores e aplicam-nas em novas situações, integrando o novo conhecimento que adquiriram com construções intelectuais pré-existentes. Na perspetiva construtivista, a aprendizagem realiza-se quando somos capazes de elaborar uma representação pessoal sobre um determinado aspeto da realidade, ou sobre um determinado conteúdo que pretendemos aprender. Claro que quando quem aprende parte desta aprendizagem, leva já as suas experiências, interesses e conhecimentos, que lhe permitam resolver a situação, adequando-a e integrando-a, construindo assim um significado próprio e pessoal. Quando este processo se verifica diz-se que o aluno aprende significativamente. Nesta abordagem da aprendizagem pretende-se oferecer aos alunos a oportunidade de ter experiências concretas e contextualizadas, que Ihes permitam procurar padrões, levantar as suas próprias questões e construir os seus próprios modelos, conceitos e estratégias (Fosnot, 1996).

\section{Portefólios na educação}

A estratégia da utilização do portefólio na educação é relativamente recente. Tal como refere Sá-Chaves (2000), o recurso ao portefólio permite aprofundar o conhecimento da relação entre o ensino e a aprendizagem e, com base nesse conhecimento, conseguir índices de qualidade mais elevados. A mesma autora afirma que o recurso ao portefólio pode ser um excelente aliado a todos quantos pretendem ver a sua prática pedagógica melhorada, já que este recurso proporciona uma ajuda incontestável na mudança e na melhoria das práticas pedagógicas, contribuindo para:

Promover o desenvolvimento reflexivo dos participantes, quer ao nível cognitivo, quer metacognitivo; Estimular o processo de enriquecimento conceptual, através do recurso às múltiplas fontes de conhecimento em presença; Estru- 
turar a organização conceptual ao nível individual, através da progressiva aferição de critérios de coerência, significado e relevância pessoal; Fundamentar os processos de reflexão para, na e sobre a ação, quer na dimensão pessoal, quer profissional; Garantir mecanismos de aprofundamento conceptual continuado, através do relacionamento em feedback entre membros das comunidades de aprendizagem; Estimular a originalidade e criatividade individuais no que se refere aos processos de intervenção educativa, aos processos de reflexão sobre ela e à sua explicitação, através de vários tipos de narrativa; Contribuir para a construção personalizada do conhecimento para, em e sobre a ação, reconhecendo-lhe a natureza dinâmica, flexível, estratégica e contextual; Permitir a regulação em tempo útil, de conflitos de etiologia diferenciada, garantindo condições de estabilidade dinâmica e de desenvolvimento progressivo da autonomia e da identidade; Facilitar os processos de auto e heteroavaliação, através da compreensão dos processos (Sá-Chaves, 2000, p. 10).

A par do que foi referido anteriormente, o portefólio pode de ser uma alternativa aos métodos de avaliação mais tradicionais, o portefólio é, como atrás contactámos, um dos mecanismos existentes na educação que dá a oportunidade ao professor de contemplar mudanças nas práticas curriculares, já que a utilização deste instrumento de avaliação implica alterações na forma como se trabalham os conteúdos e, consequentemente, no modo como decorrem os processos de ensino-aprendizagem. Ademais, a sua utilização é normalmente encarada como uma excelente forma de aprender e de ensinar, já que obriga ao envolvimento dos alunos na construção dos seus saberes, o trabalho em equipa é estimulado, implica os alunos na avaliação dos resultados e responsabiliza-os pela sua própria aprendizagem, contribuindo para a obtenção de um dos principais desígnios de qualquer sistema educativo que é a de formar indivíduos capazes de aprender de forma autónoma ao longo da vida (Farr \& Tone, 1998).

\subsection{O uso efectivo do Portefólio}

O professor, ao delinear o processo de ensino, deverá considerar o triângulo didático reinterpretado (Trindade, 2003) citado por Prazeres \& Casanova (2015), em que os alunos nos seus contextos possuem um papel predominante. Como prosseguem as autoras, deverá o professor planificar as estratégias de ensino tendo em conta os estilos de aprendizagem que os alunos acarretam, selecionar e definir quais são os conteúdos que os alunos devem aprender e, ainda, definir quais as técnicas que proporcionam aprendizagem profunda conducentes à transferência de aprendizagem. No que concerne à avaliação, afirmam as autoras, citando Black e Wiliam (2009), que a mesma tem de se colocar ao serviço da aprendizagem do aluno, encontrando-se em permanente reorganização dialógica entre professores e alunos. 
Assim, antes da utilização do instrumento, foram disponibilizados aos alunos os objetivos traçados. De maneira a que conseguissem, desde logo, perceber o alcance da utilização do portefólio, os alunos foram informados que no final era pretendido que eles fossem capazes de:

- Desenvolver a capacidade de definir necessidades, interesses, objetivos e estratégias.

- Sistematizar aprendizagens.

- Selecionar estratégias mais adequadas ao seu estilo cognitivo.

- Desenvolver a capacidade de autoavaliação.

- Desenvolver a autonomia.

- Desenvolver a iniciativa.

- Consciencializar-se da própria responsabilidade no processo de aprendizagem

- Refletir acerca do percurso formativo.

Do mesmo modo foram definidos e partilhados com os alunos os critérios de avaliação selecionados e que agrupámos em torno de quatro categorias:

1. seleção: referenciação, pertinência, diversidade e representatividade dos documentos escolhidos;

2. reflexão: qualidade, correção e rigor das justificações e dos comentários escritos;

3. organização: estrutura, organização e apresentação dadas ao portefólio;

4. apresentação oral do portefólio.

Deixamos para último um tópico que ainda (e propositadamente) não referimos: o feedback. Deixamos que fosse o último ponto a ser mencionado neste trabalho, porque o consideramos demasiadamente importante e quisemos que ele se destacasse por algum motivo. No processo educativo, como na vida, o feedback faz-nos recentrar no processo, faz-nos sentir importantes no processo e possibilita-nos melhorar se nos for permitido. Um dos grandes problemas do sistema de ensino tradicional, é que ele apenas proporcionava feedback ao aluno no final do processo através de uma nota final que o quantifica. Não acreditamos em sistemas de ensino e em práticas pedagógicas que não valorizam o processo de ensino-aprendizagem e não cremos que esses sistemas ajudem o aluno a subsistir na sociedade quando forem desafiados a transferir aprendizagens em contextos reais e não controlados, diferentes de quando estão num ambiente de aprendizagem seguro, como a escola.

O feedback eficaz é descrito por Brookhart (2008), citado por Fonseca et al. (2015), contemplando duas dimensões: a cognitiva e a motivacional. Por um 
lado, a dimensão cognitiva no que respeita ao fornecimento de informações necessárias aos alunos para poderem compreender "em que ponto se encontram na sua aprendizagem e o que têm de fazer a seguir" (p. 2); a dimensão motivacional diz respeito ao desenvolvimento, nos alunos, da "sensação de que têm controlo sobre sua própria aprendizagem" (p. 2).

A literatura aponta no sentido de considerar as estratégias de feedback como um elemento muito significativo na promoção da relação entre professores e alunos, bem como no envolvimento académico destes e no seu desempenho e autorregulação de aprendizagens (Black \& Wiliam, 1998; Hattie, 2009).

Como apontam os trabalhos de Brookhart (2008) e Shute (2008), sempre que o feedback fornece diretrizes claras que ajudam os alunos a ajustar a sua aprendizagem, há um impacto maior no desempenho escolar, maior probabilidade de que os alunos se envolvam nas tarefas que lhes são solicitadas e assiste-se à sua perseverança na consecução dos objetivos de aprendizagem.

\section{Conclusões}

Os desafios com que as instituições de ensino e todos os agentes educativos se debatem de uma forma regular levam-nos a concluir que o papel do professor no processo de ensino e aprendizagem é um papel dinâmico e que o obriga a uma constante reposicionamento e imenso debate interior em relação às práticas e modelos pedagógicos. Do mesmo modo, o aluno procura que o sistema educativo Ihe dê respostas eficazes e que o prepare para o mundo "real", munindo-o de competências que o façam singrar. É por isto que acreditamos em sistemas de ensino que contemplem alunos que desenvolvam uma autonomia e um controlo sobre a sua própria aprendizagem, tornando-os agentes do seu próprio conhecimento. É essencial que os professores se esforcem por compreender de que forma os alunos se apropriam do conhecimento, que lhes forneçam um contínuo feedback da aquisição de competências que pretendem que desenvolvam e que, com isso, contribuam de uma maneira muito positiva para uma autorregulação da aprendizagem. Urge, também, que as estratégias educativas sejam repensadas de uma maneira sistemática e que se debatam intervenções que promovam e incentivem ao compromisso do aluno com a aprendizagem no sentido restrito e com a escola no sentido global.

Pese embora este artigo tenha tinha como mote a exaltação do uso do portfólio, na medida em que consideramos que, dadas as suas potencialidades, poderá ser um excelente meio ao serviço do ensino, convém salvaguardar que estão reunidas as condições aquando da decisão do seu uso, nomeadamente o facto de se ter consciência que os portfólios não são padronizados, poderão não ser viáveis para avaliação em grande escala, nomeadamente por causa do excessivo tempo 
que requerem para uma avaliação bem sucedida e poderão ser potencialmente tendenciosos. Abaixo, elencamos algumas das desvantagens acima referidas que foram sistematizadas por Venn (2000):

- Requiring extra time to plan an assessment system and conduct the assessment.

- Gathering all of the necessary data and work samples can make portfolios bulky and difficult to manage.

- Developing a systematic and deliberate management system is difficult, but this step is necessary in order to make portfolios more than a random collection of student work.

- Scoring portfolios involves the extensive use of subjective evaluation procedures such as rating scales and professional judgment, and this limits reliability.

- Scheduling individual portfolio conferences is difficulty and the length of each conference may interfere with other instructional activities. (p. 538)

O processo de ensino-aprendizagem é, como sabemos, um dos processos mais complexos, sendo, contudo, para nós, enquanto agentes da educação, um processo que nos fascina. A incessante procura que fazemos para encontrar o método mais adequando para ensinar ou o instrumento mais justo para avaliar marca a nossa prática que todos os anos letivos se altera em consonância com os novos alunos que recebemos e com a conjuntura que influencia, sempre, o processo de ensinar, mas também o processo de aprender.

\section{Referencias bibliográficas}

Abrami, P. C., \& Barrett, H. (2005). Directions for research and development on electronic portfolios. Canadian Journal of Learning and Technology, 31(3). https://doi.org/10.21432/ T2RK5K

Arter, J.A., \& Spandel, V. (1992). Using portfolios of student work in instruction and assessment. Educational Measurement: Issues \& Practice, 11(1), 36-44. https://doi. org/10.1111/j.1745-3992.1992.tb00230.x

Barreira, C., Bidarra, G., Monteiro, F., VazRebelo, P., \& Alferes, V. (2017). Avaliação das aprendizagens no ensino superior. Percesōes de professores e estudantes nas universidades portuguesas. Revista Iberoamericana de Educación Superior, VIII(21),24-36.
Bidarra, M.G., \& Festas, M.I. (2005).

Construtivismo (s): Implicações e interpretações educativas. Revista Portuguesa de Pedagogia, 39 (2), 177-195. https://bit. $\underline{\mathrm{l} / 3 \mathrm{aJNy} 2 \mathrm{~B}}$

Black, P., \& Wiliam, D.. (2009). Developing the theory of formative assessment. Educational Assessment Evaluation and Accountability. 21. 5-31. https://doi.org/10.1007/s11092-0089068-5.

Black, P., \& Wiliam, D. (1998). Inside the black box: Raising standards through classroom assessment. PDK International. https://doi. org/10.1177/003172171009200119

Bloom, B. S. (1969). Some theoretical issues relating to educational evaluation. In R. W. 
Tyler (Ed.), Educational evaluation: New roles, new means (National Society for the Study of Education Yearbook, Vol. 68, Part 2, pp. 2650). University of Chicago Press.

Bloom, B. S., Hastings, J. T., \& Madaus, G. F. (1971). Handbook on the formative and summative evaluation of student learning. McGraw-Hill.

Brookhart, S. (2008). How to give effective feedback to your students. Association for Supervision and Curriculum Development.

Brown, H. D. (2001). Teaching by Principles: an interactive approach to language pedagogy. Longman

Brown, K. G., \& Gerhardt, M. W. (2002). Formative evaluation: An integrative practice model and case study.Personnel Psychology,55(4), 951-983. https://doi. org/10.1111/j.1744-6570.2002.tb00137.x

Butler, P. (2006). A review of the literature on portfolios and electronic portfolios. Massey University College of Education. https://bit. ly/3xuSmm2

Chang, C. (2001). Construction and evaluation of a web-based learning portfolio system: An electronic assessment tool. Innovations in Education and Teaching International, 38(2), 144-155.

Farr, R. C., \& Tone, B. (1998). Portfolio and Performance Assessment: Helping Students Evaluate their Progress as Readers and Writers. Harcourt Brace College Publishers.

Fernandes, Domingos \& Borralho, António \& Barreira, Carlos. (2015). Avaliação, Ensino E Aprendizagem No Ensino Superior Em Portugal E No Brasil: Realidades e Perspetivas (Volume I)

Fonseca, J. et al. (2015). Feedback na prática letiva: Uma oficina de formação de professores. Rev. Port. de Educação, vol.28, no.1, p.171-199. ISSN 0871-9187

Formosinho, J. (1992). O Dilema Organizacional da Escola de Massas. Revista Portuguesa de Educação.

Fosnot, C. T. (1996). Construtivismo: uma teoria psicológica da aprendizagem. In C.
T. Fosnot (Ed.) Construtivismo e educação. Teoria, perspectivas e prática. Instituto Piaget: Horizontes Pedagógicos.

Gates, B. (1995). Rumo ao Futuro. McGrawHill.

Hattie, J., \& Timperley, H. (2007). The power of feedback. Review of Educational Research, 77(1), 81-112.

Hattie, J. (2009). Visible learning: A synthesis of over 800 meta-analyses relating to achievement. Routledge.

Leite, E. (1995). O Professor Aprendiz - Criar o Futuro. Programa Europeu Petra II, Acção II. Ministério da Educação/ Departamento do Ensino Secundário.

Lopes, J., \& Silva, H. (2010). O professor far. a diferença. $\mathrm{Na}$ aprendizagem dos alunos. $\mathrm{Na}$ realização escolar dos alunos. No sucesso dos alunos. Lidel.

Lopes, J., \& Silva, H. (2012). 50 Técnicas de avaliação formativa.Lidel.

Matos,J.A. (2006). Trajectórias Interdisciplinares. Uma Aplicação Multimédia sobre o Alto Douro. http://hdl.handle.net/10216/64122

Moran, J. M. (2000). Novas Tecnologias e Mediação Pedagógica. Papirus.

Morin, E. (2002). Reformar o Pensamento, A Cabeça Bem Feita. Instituto Piaget.

Neves, A., \& Barbosa, J. (2006). Fantasmas, mitos e ritos da avaliação das aprendizagens. Revista Portuguesa de Pedagogia, 3, 219235. https://doi.org/10.14195/16478614_40-3\%25x

Nunan, D. (1995). Language Teaching Methodology: a textbook for teachers. Phoenix : ELT.

Perrenoud, P. (1998). From formative evaluation to a controlled regulation of learning processes. Towards a wider conceptual field. Assessment in Education: Principles, Policy \& Practice,5(1), 85-102. https://doi.org/10.1080/0969595980050105 
Ponte, J., \& Serrazina, L. (1998). As

Novas Tecnologias na Formação Inicial de

Professores. Lisboa : Ministério da Educação,

Departamento de Avaliação, Prospectiva e

Planeamento. ISBN 972-614-343-8

Prator, C.H. (1979). Cornerstones of method and names for the profession. In M. M.

Murcia (1991). Teaching English as a Second or

Foreign Language. Newbury House Publishers.

Prazeres, M., \& Casanova, M. (2015) . A Avaliação como Promoção da Aprendizagem dos Alunos. In Teresa Estrela et al. Atas do XXII Congresso da AFIRSE: Diversidade e Complexidade da Avaliação em Educaşão e Formação. Educa/Afirse (pp.1282-1290).

Sá-Chaves, I. (2000). Portefólios Reflexivos. Estratégia de Formação e de Supervisão. Universidade de Aveiro.

Santos, M. (s/data). Um olhar sobre o conceito de comunidades de prática. http://www.educ.fc.ul. $\mathrm{pt} /$ docentes/jfmatos/mestrados/Santos CdP 2002.pdf
Shute, V. J. (2008). Focus on formative feedback. Review of Educational Research, 78(1), 153-189. https://doi. org/10.3102/0034654307313795

Silva, H., \& Lopes, J. (2015). Eu, professor, pergunto. 20 respostas sobre planificação do ensinoaprendizagem, estratégias de ensino e avaliação (Vol. 1). PACTOR.

Trindade, V. M. (2003). Uma Perspectiva Didáctica para o Ensino da Ciências. In Org. Costa, P., Chouriço, J., Mendes, P. Neto, A. e Nico, A.. Didácticas e Metodologias de Educação. Percursos e Desafios. Universidade de Évora. Departamento de Pedagogia e Educação.

Venn, J. J. (2000). Assessing students with special needs (2nd ed.). Merrill

Wiliam, D. (2006). Formative assessment: Getting the focus right. Educational Assessment, 11(3 \& 4), 283-289. https://doi.org/10.1207/ s15326977ea1103\&4 7 